(BDI-II), PROs assessing nutrition and fatigue], body composition was analyzed using densitometry (iDXA Lunar) and bioelectric impedance (BIA2000-M), and patients provided blood for routine laboratory analysis and biobanking. Normality of data was tested and inter-group analysis performed with 2-way ANOVA and intra-group analysis by Friedmann's test with Dunn's post hoc test.

Results: 27 IIM patients (22 female/5 male, 10 DM/12 PM/5 IMNM (immune mediated necrotizing myopathy), median of age 58.0 and disease duration 7.0 years) were recruited into the intervention group (IG) and 27 patients into the control group (CG) (24 female/3 male, 13 DM/12 PM/2 IMNM, median of age 56.5 and disease duration 4.7 years). Compared to observed statistically significant deterioration in $C G$ over the period of months $0-6$, we found statistically significant improvement in FI-2, MMT8, HAQ, BDI-II (Table 1). Only numerical improvement in IG compared to numerical deterioration in CG, which has not reached statistical significance, was observed in SF-36 and fatigue PROs.

\begin{tabular}{|c|c|c|c|c|c|}
\hline \multirow{2}{*}{$\begin{array}{c}\text { Parameter } \\
\text { (unit) }\end{array}$} & \multirow{2}{*}{$\begin{array}{l}\text { Intervention group } \\
\text { Mean } \pm \mathrm{SEM}\end{array}$} & \multirow{2}{*}{$\begin{array}{l}\text { Control group } \\
\text { Mean } \pm \mathrm{SEM}\end{array}$} & \multicolumn{2}{|c|}{ Intra-group analysis (Friedmann+Dunn) } & \multirow{2}{*}{$\begin{array}{c}\text { Inter-group } \\
\text { analysis (2WA) }\end{array}$} \\
\hline & & & Interevention gr. & & \\
\hline \multirow{3}{*}{ MMT-8 } & & $m 0: 64.2 \pm 2.3$ & $m 0-m 3: p<0.001$ & m0-m3: p<0.01 & \multirow{3}{*}{$p<0.0001$} \\
\hline & $\mathrm{m} 3: 60.7 \pm 2.4$ & $\mathrm{~m} 3: 58.4 \pm 2.0$ & $m 3-m 6: p<0.01$ & $m 3-m 6: p=N S$ & \\
\hline & $m 6: 69.1 \pm 1.9$ & $m 6: 55.3 \pm 2.0$ & $m 0-m 6: p<0.001$ & $m 0-m 6: p<0.001$ & \\
\hline \multirow{3}{*}{$\mathrm{Fl}-2(\%)$} & $\mathrm{m} 0: 30.0 \pm 4.4$ & $m 0: 40.2 \pm 5.9$ & $m 0-m 3: p<0.001$ & $\mathrm{~m} 0-\mathrm{m} 3: \mathrm{p}=\mathrm{NS}$ & \multirow{3}{*}{$p<0.0001$} \\
\hline & $\mathrm{m} 3: 46.9 \pm 4.7$ & $\mathrm{~m} 3: 30.8 \pm 4.9$ & $m 3-m 6: p<0.001$ & $m 3-m 6: p=N S$ & \\
\hline & $m 6: 70.6 \pm 4.9$ & $m 6: 28.7 \pm 4.5$ & $m 0-m 6: p<0.001$ & $m 0-m 6: p<0.01$ & \\
\hline \multirow{3}{*}{ HAQ } & $\mathrm{m} 0: 0.91 \pm 0.16$ & $\mathrm{~m} 0: 1.25 \pm 0.17$ & $m 0-m 3: p=N S$ & $m 0-m 3: p=N S$ & \multirow{3}{*}{$p=0.0012$} \\
\hline & $\mathrm{m} 3: 0.69 \pm 0.14$ & $\mathrm{~m} 3: 1.33 \pm 0.18$ & $m 3-m 6: p=N S$ & $m 3-m 6: p=N S$ & \\
\hline & $m 6: 0.56 \pm 0.11$ & $\mathrm{~m} 6: 1.27 \pm 0.19$ & mo-m6: $p<0.001$ & $m 0-m 6: p=N S$ & \\
\hline \multirow{3}{*}{ BDI-II } & $\mathrm{m} 0: 11.9 \pm 2.1$ & $\mathrm{~m} 0: 13.3 \pm 1.5$ & $\mathrm{~m} 0-\mathrm{m} 3 \cdot \mathrm{p}=\mathrm{NS}$ & $\mathrm{m} 0-\mathrm{m} 3: \mathrm{p}=\mathrm{NS}$ & \multirow{3}{*}{$\mathrm{p}=0.0186$} \\
\hline & & & $m 3-m 6: p=N S$ & $m 3-m 6: p=N S$ & \\
\hline & $\begin{array}{ll}m 6: 8.9 \pm 1.5 \\
m 6.1 .19\end{array}$ & $\mathrm{~m} 6: 14.6 \pm 1.4$ & $m 0-m 6: p<0.05$ & $\mathrm{~m} 0-\mathrm{m} 6: \mathrm{p}=\mathrm{NS}$ & \\
\hline
\end{tabular}

Conclusions: Our intensive 24-week physiotherapy programme led to a significant improvement in muscle strength, endurance, function and depression, which was clinically meaningful in a substantial proportion of patients.

Acknowledgements: Supported by AZV-16-33574A

Disclosure of Interest: None declared

DOI: 10.1136/annrheumdis-2017-eular.4910

\section{AB0654 AN ULTRASOUND ASSESSMENT OF THE HAND AND WRIST IN EGYPTIAN PATIENTS WITH SYSTEMIC SCLEROSIS}

M. Hassanien, S. Rashad, A. Aljohee. Rheumatology Department, assuit, Egyp

Background: Systemic sclerosis (SSc, scleroderma) is a systemic disease characterized by fibrosis, progressive vascular obliteration and the production of autoantibodies.Ultrasound (US) imaging has advantages of simplicity, low cost, noninvasiveness, real-time capability and portability compared with traditional EDX. US imaging also offers high temporal and spatial resolutions, and can potentially provide dynamic anatomical information regarding local structures and kinesiology, few studies evaluate joint and tendons involvement in SSc by US the incidence and abnormality are differs among studies

Objectives: describe and determine possible correlations between US abnormalities of hand and wrist and clinical findings in SSc Egyptian patients

Methods: Randomly selected 50 Egyptian SSc patients were enrolled in the study in outpatient clinic Rheumatology department, Assuit university hospital. Clinical examination and US evaluation (Gray Scale and Power Doppler (PD)) of the tendons and joints of hands and wrists, were performed bilaterally on both dorsal and palmar sides. Joint assessment was performed on all MCP and PIP joints as well as the wrist and tendon assessment included finger flexors and extensors, wrist extensors and flexors. The scoring for synovitis was based on the EULAR/ OMERACT scoring system

Results: Within the cohort, $100 \%$ of the patients were female; mean age and range disease duration were $40.93 \pm 11.71(20-61)$ years and $8.13 \pm 5.56$ (0.5-25)years, respectively. Modified Rodnan Skin Score mean and range were $23.17 \pm 9.14(13-43), 22$ patients were diffuse type (44\%) and 28 patients were limited type (56\%), $67 \%$ had inflammatory arthralgia as reported by patients. US examination revealed abnormalities in $76 \%$ of all cases. Synovitis was present in $74 \%$ of patients ( $22 \%$ wrist; $15 \%$ MCP/PIP, $63 \%$ both); with a grade 2 and 3 synovitis in $29 \%$ cases. The grade 2 or 3 synovitis was associated with a higher age $(p<0.05)$ and disease duration $(p<0.03)$ but not with inflammatory markers (CRP, ESR) and C3 nor with anti-CCP antibodies.

Furthermore, patients with US proven grade 2 or 3 synovitis had interstitial lung disease involvement (ILD) (74\%) and elevated systolic pulmonary artery pressure $>35 \mathrm{mmHg}$ in (46\%). Grade 1 synovitis did not correlate with tenderness or joint swelling. Synovitis using PD abnormalities were present in $20 \%$ patients, but only a $25 \%$ had concomitant clinical synovitis. Bone erosions $(>1 \mathrm{~mm})$ were observed in $15 \%$, which located at the second and third MC heads and at the styloid process of the ulna. The presence of bone erosions was associated with the presence of grade 2 or 3 synovitis. US identified tenosynovitis in $40 \%$ of patients, involving the wrist extensors in the vast majority of cases $(70 \%)$, especially of the 2nd extensor compartment

Conclusions: US examination detects significant synovitis of the hand and wrist in the absence of clinical findings, and reveals structural damage in a number of patients. The grade 2 or 3 synovitis was associated with a higher age, and ILD involvement. Tendon involvement is frequent with specific pattern of wrist extensors. Thus, US seems to be a valuable tool to identify subclinical joint manifestations in Egyptian SSc patients.
Disclosure of Interest: None declared

DOI: 10.1136/annrheumdis-2017-eular.1101

\section{AB0655 ULTRASOUND B-LINES IN THE EVALUATION OF INTERSTITIAL LUNG DISEASE IN PATIENTS WITH SYSTEMIC SCLEROSIS: CUT-OFF POINT DEFINITION FOR THE PRESENCE OF PULMONARY FIBROSIS}

M. Tardella ${ }^{1}$, M. Di Carlo ${ }^{1}$, M. Carotti ${ }^{2}$, E. Filippucci ${ }^{1}$, W. Grassi ${ }^{1}$, F. Salaffi ${ }^{1}$.

${ }^{1}$ Rheumatology Department, Università Politecnica delle Marche, Jesi;

${ }^{2}$ Radiology Department, Università Politecnica delle Marche, Ancona, Italy

Background: Although in Systemic Sclerosis (SSc) the cutaneous manifestations are the most noticeable features, lung involvement guides the disease prognosis. High resolution computed tomography (HRCT) is the mainstay for the detection of the interstitial lung disease (ILD). However, exposure to ionising radiation is a major limitation for the repetition of this examination. Over the last years lung ultrasound (US) gained an intriguing potential in the evaluation of several pulmonary condition, and in previous studies was found a significant correlation between US score and HRCT score [1]

Objectives: To establish the cut-off point of the US B-lines number for detecting the presence of SSc-ILD.

Methods: Patients with SSc-ILD underwent chest HRCT, lung US, pulmonary function test, and clinical assessment. Exclusion criteria were represented by the presence of a coexisting congestive heart failure and other lung or pleural diseases (i.e., pneumonia, pleural effusion). HRCT were scored for the presence of ILD by an experienced radiologist, in accordance with the Warrick method. US assessment was performed by a US skilled rheumatologist, blinded to the HRCT results, and included the bilateral evaluation of 14 lung intercostal spaces (LIS) [2]. In each LIS the number of B-lines was recorded and summed. To test discriminant validity we used the receiver operating characteristic (ROC) curve analysis applying a Warrick score of 7 as external criterion for the presence of SSc-ILD.

Results: Forty patients completed the study. The US B-lines number and the Warrick score confirmed an excellent correlation (Spearman's rho: 0.819 , $p<0.001)$. The ROC curve analysis revealed that the presence of 10 US B-lines is the cut-off point with the greatest positive likelihood ratio (12.52) for the presence of SSc-ILD (Table).

\begin{tabular}{lcccccccc}
\hline $\begin{array}{l}\text { US B-lines } \\
\text { number }\end{array}$ & Sensitivity & $95 \% \mathrm{Cl}$ & Specificity & $95 \% \mathrm{Cl}$ & $+\mathrm{LR}$ & $95 \% \mathrm{Cl}$ & $-\mathrm{LR}$ & $95 \% \mathrm{Cl}$ \\
\hline$\geq 2$ & 100.00 & $87.2-100.0$ & 0.00 & $0.0-24.7$ & 1.00 & & & \\
$>2$ & 100.00 & $87.2-100.0$ & 15.38 & $1.9-45.4$ & 1.18 & $0.3-4.2$ & 0.00 & \\
$>3$ & 100.00 & $87.2-100.0$ & 46.15 & $19.2-74.9$ & 1.86 & $1.0-3.3$ & 0.00 & \\
$>4$ & 100.00 & $87.2-100.0$ & 61.54 & $31.6-86.1$ & 2.60 & $1.7-4.0$ & 0.00 & \\
$>5$ & 100.00 & $87.2-100.0$ & 69.23 & $38.6-90.9$ & 3.25 & $2.3-4.7$ & 0.00 & \\
$>8$ & 100.00 & $87.2-100.0$ & 76.92 & $46.2-95.0$ & 4.33 & $3.2-5.8$ & 0.00 & \\
$>9$ & 96.30 & $81.0-99.9$ & 84.62 & $54.6-98.1$ & 6.26 & $4.9-8.0$ & 0.044 & $0.004-0.4$ \\
$>10$ & 96.30 & $81.0-99.9$ & 92.31 & $64.0-99.8$ & 12.52 & $10.5-14.9$ & 0.040 & $0.003-0.6$ \\
$>11$ & 92.59 & $75.7-99.1$ & 92.31 & $64.0-99.8$ & 12.04 & $10.0-14.6$ & 0.080 & $0.008-0.8$ \\
$>14$ & 88.89 & $70.8-97.6$ & 92.31 & $64.0-99.8$ & 11.56 & $9.4-14.2$ & 0.12 & $0.01-1.0$ \\
$>15$ & 85.19 & $66.3-95.8$ & 92.31 & $64.0-99.8$ & 11.07 & $8.9-13.8$ & 0.16 & $0.02-1.3$ \\
$>16$ & 81.48 & $61.9-93.7$ & 92.31 & $64.0-99.8$ & 10.59 & $8.3-13.4$ & 0.20 & $0.03-1.5$ \\
$>19$ & 70.37 & $49.8-86.2$ & 92.31 & $64.0-99.8$ & 9.15 & $6.8-12.2$ & 0.32 & $0.04-2.3$ \\
$>20$ & 59.26 & $38.8-77.6$ & 92.31 & $64.0-99.8$ & 7.70 & $5.4-10.9$ & 0.44 & $0.06-3.1$ \\
$>21$ & 55.56 & $35.3-74.5$ & 92.31 & $64.0-99.8$ & 7.22 & $5.0-10.5$ & 0.48 & $0.07-3.3$ \\
$>22$ & 51.85 & $31.9-71.3$ & 92.31 & $64.0-99.8$ & 6.74 & $4.5-10.0$ & 0.52 & $0.08-3.6$ \\
\hline
\end{tabular}

Conclusions: The detection of $10 \mathrm{~B}$-lines is highly predictive for the HRCT presence of SSc-ILD. In SSc patients, a lung US follow-up can optimize the employment of chest HRCT.

Disclosure of Interest: None declared

DOI: 10.1136/annrheumdis-2017-eular.6828

\section{AB0656 NAILFOLD VIDEOCAPILLAROSCOPY FEATURES OF PATIENTS WITH ANTISYNTHETASE SYNDROME}

M. Sebastiani ${ }^{1}$, V. Codullo ${ }^{2}$, A. Manfredi ${ }^{1}$, A. Selva O'Callaghan ${ }^{3}$, F. Furini $^{4}$, A. Iuliano ${ }^{5}, A$. Schwarting ${ }^{6,7}$, L. Cavagna ${ }^{2}, K$. Triantafyllias ${ }^{7}$ on behalf of NASCAR Study Group. 'Policlinico di Modena, University of Modena and Reggio Emilia, Modena; ${ }^{2}$ University of Pavia, Pavia, Italy; ${ }^{3}$ Universidad Autonoma de Barcelona, Barcelona, Spain; ${ }^{4}$ University of Ferrara, Ferrara; ${ }^{5}$ Azienda Ospedaliera San Camillo-Forlanini, Roma, Italy; ${ }^{6}$ University Mainz, Mainz; ${ }^{7}$ ACURA Rheumatology Clinics, Bad Kreuznach, Germany

Background: Antisynthetase syndrome (ASSD) is an autoimmune disease characterized by the clinical triad arthritis, myositis, and interstitial lung disease (ILD). As in inflammatory myopathies, nailfold videocapillaroscopy (NVC) alterations have been sporadically described also in ASSD patients, but no elucidating data are available.

Objectives: To investigate the possible specific NVC features of ASSD patients. Methods: Within the framework of a multicenter study, we retrospectively analyzed NVC images of ASSD patients, after excluding patients with overlap syndrome with systemic sclerosis. Two operators in a blind manner re-evaluated all 